\title{
Well-ordered Nanostructure Formation of Wholly Aromatic Poly(amic acid)s in Spin-casted Thin Films
}

\author{
Ling Gao, Koei Azuma, Yuta Kushima, Kenta Okuhara, Alvin Chandra, and Teruaki Hayakawa \\ Department of Organic and Polymeric Materials, Tokyo Institute of Technology, 2-12-1- \\ S8-36 Ookayama, Meguro-ku, Tokyo, 152-8552, Japan
}

Keywords: Poly(amic acid), Wholly Aromatic Polymer, Spin-cast, Thin Film, Block Copolymer, Nanostructure

\section{Introduction}

Ordered mesoporous polymer films with a high thermal stability and good mechanical strength have become increasingly important in a variety of advanced technical applications. The block copolymer (BCP) template-assisted approach is a simple way to fabricate ordered nanostructures based on the microphase separation of experimentally tunable BCPs. As such, numerous studies regarding mesoporous bulk films [1,2] based on phenolic resin precursors using BCPs as a soft-template have been reported. However, these studies were limited to olefin type BCPs for the formation of the ordered nanostructures, making it difficult to obtain high performance films. To circumvent this problem, aromatic polyimides (PIs) can be made using a BCP softtemplate to exploit their increased thermal and chemical stabilities, and remarkable mechanical and electrical properties. However, as far as we know, there have been few reports regarding mesoporous polyimide films as polyimides are conventionally synthesized via a step-growth polymerization, thus making control of the nanostructure formation difficult.

In a previous study by our group, we reported a novel technique that combined wholly aromatic condensation oligomers with olefin type BCPs to create the desired ordered mesoporous polymer films. However, with this newly demonstrated approach, we were only able to obtain relatively thick films with a thickness of several micrometers [3]. The main challenge to obtaining high performance thin films, which are necessary for industrial applications, is the precise control of the BCP morphologies in thin films. Herein, the nanostructure formation of aromatic poly(amic acid)s (PAAs) which can be used as a polyimide precursor in spin-casted thin films was studied in detail. (Scheme 1).

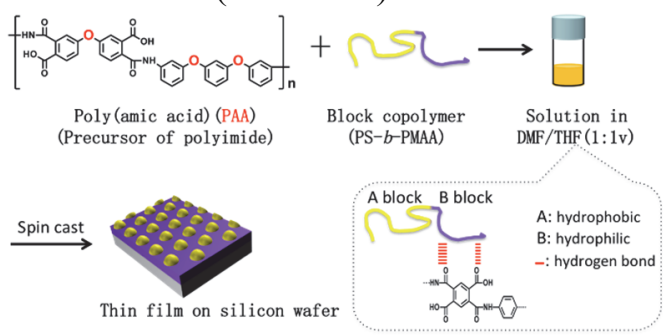

Scheme 1.

\section{Experimental}

2.1. Synthesis of the copolymer

Polystyrene-block-poly(t-butyl methacrylate) (PS- $b$-P $t$ BuMA $)$ copolymer $\left(\mathrm{M}_{\mathrm{n}, \mathrm{PS}}=20 \mathrm{~kg} / \mathrm{mol}\right.$, $\mathrm{M}_{\mathrm{n}, \mathrm{PtBuMA}}=8 \mathrm{~kg} / \mathrm{mol}$, PDI $=1.05,71 \mathrm{wt} \% \mathrm{PS}, 29$ $\mathrm{wt} \% \quad \mathrm{P} t \mathrm{BuMA})$ was synthesized via anionic polymerization. Polystyrene-blockpoly(methacrylic acid) (PS- $b$-PMAA, $80 \mathrm{wt} \%$ PS, $20 \mathrm{wt} \%$ PMAA) was subsequently obtained by the hydrolysis of the PS- $b$-P $t$ BuMA copolymer. Poly(amic acid) (PAA) was prepared by the polymerization of 5,5'-oxybis(isobenzofuran-1,3dione) and 3,3'-(1,3-phenylenebis(oxy))dianiline using N,N-dimethylacetamide (DMAc) as a solvent. The inherent viscosity of the PAA solution was $0.35 \mathrm{gdL}^{-1}$ (at a concentration of 0.5 $\operatorname{gdL}^{-1}$ at $30^{\circ} \mathrm{C}$, Ostwald viscometer).

\subsection{Preparation of the composite polymer} spin-coated thin film

Samples with different weight ratios of PS- $b$ PMAA and PAA were dissolved and stirred overnight in a co-solvent of N,Ndimethylformamide (DMF) and tetrahydrofuran (THF) $(1: 1, \mathrm{v} / \mathrm{v})$. Thin films were prepared by spin-casting polymer solutions onto silicon

Received April 1, 2016

Accepted May 11, 2016 
wafers.

2.3. Preparation of the composite polymer thin film with cross-linker

The resol precursor was synthesized in a previous report [4] and used without further purification as the cross-linker. PS- $b$-PMAA, PAA, resol precursor and one drop of methanesulfonic acid were dissolved in a cosolvent of DMF/THF $(1: 1, \mathrm{v} / \mathrm{v})$ with a weight ratio of PS- $b$-PMAA/PAA/resol precursor of $35 / 55 / 10$. After leaving the mixture to stir overnight, the homogeneous solution $(3 \mathrm{wt} \%$ in solution) was spin-casted onto silicon wafers to obtain the thin films. Further curing was performed at the following temperature profile: $50{ }^{\circ} \mathrm{C}$ for $7 \mathrm{~h}, 60{ }^{\circ} \mathrm{C}$ for $12 \mathrm{~h}, 80{ }^{\circ} \mathrm{C}$ for $7 \mathrm{~h}$, $100^{\circ} \mathrm{C}$ for $12 \mathrm{~h}, 120^{\circ} \mathrm{C}$ for $30 \mathrm{~min}, 150^{\circ} \mathrm{C}$ for 30 min under vacuum.

\subsection{Measurement}

The molecular weights were determined by gel permeation chromatography (GPC) (ShowaDenko GPC-101 (Carrier solvent; THF)). The thermal decomposition behavior was investigated with an SII EXSTAR TG/DTA 7300 thermogravimetric analyzer (TGA) under nitrogen flow. The sample used in the TGA analysis was initially dried in a vacuum oven at $100{ }^{\circ} \mathrm{C}$ for 72 hours. Immediately before the measurement, the sample was heated to $100{ }^{\circ} \mathrm{C}$ at $1{ }^{\circ} \mathrm{C} \mathrm{min}^{-1}$ from room temperature and the temperature was held constant for $1 \mathrm{~h}$ before being returned to room temperature again for analysis. The sample was heated from room temperature at a rate of $10{ }^{\circ} \mathrm{C} \mathrm{min}^{-1}$ for the TGA analysis. Fourier Transform-Infrared (IR) Spectroscopy was taken with a JASCO FT/IR4100 plus spectrophotometer. Thin Film thicknesses were determined through Filmetrics Filmeasure F20. The height and phase images of the thin films were obtained by atomic force microscopy (AFM) (SII SPA400).

\section{Results and Discussion}

3.1. Comparison between morphology of composite polymer spin-coated thin film

PS- $b$-PMAA and PAA were dissolved in a cosolvent of DMF/THF $(1: 1, \mathrm{v} / \mathrm{v})$ with weight ratios of PS- $b$-PMAA/PAA of $35 / 65,45 / 55$, and $55 / 45$, respectively. These composite polymer solutions $(1 \mathrm{wt} \%)$ were spin-casted onto silicon wafers at a speed of $3000 \mathrm{rpm}$ for $40 \mathrm{~s}$ to yield thin films with a thickness around $30 \mathrm{~nm}$. The AFM height images of the composite polymers (PS- $b$ PMAA/PAA) with different weight ratios are shown in Figure 1. AFM analyses showed that an irregular structure (Figure 1(a, e)) is formed in the spin-casted pure block copolymer thin film. In contrast, well-ordered spherical domains were seen clearly in the thin films spin-casted with the polymer composites. It should be noted that certain areas of the thin film containing defects were also obtained as shown in Figure $1(\mathrm{~h}, \mathrm{i})$.

Despite the increase in the ratio of PAA to PS$b$-PMAA, no change in the morphology of the thin films could be observed. This is likely a result of the deposition of the micelle structures formed by the BCP composite during solvent evaporation. When dissolved in a co-solvent of $\mathrm{DMF} / \mathrm{THF}$, the BCP composite was observed to selectively form only micelle structures in solution. Hence, the composition ratios of the BCP composite did not affect the nanostructure formed on the substrate and only a spherical structure was observed. The darker areas in the AFM images are due to the strong interaction between the polymer and the substrate.

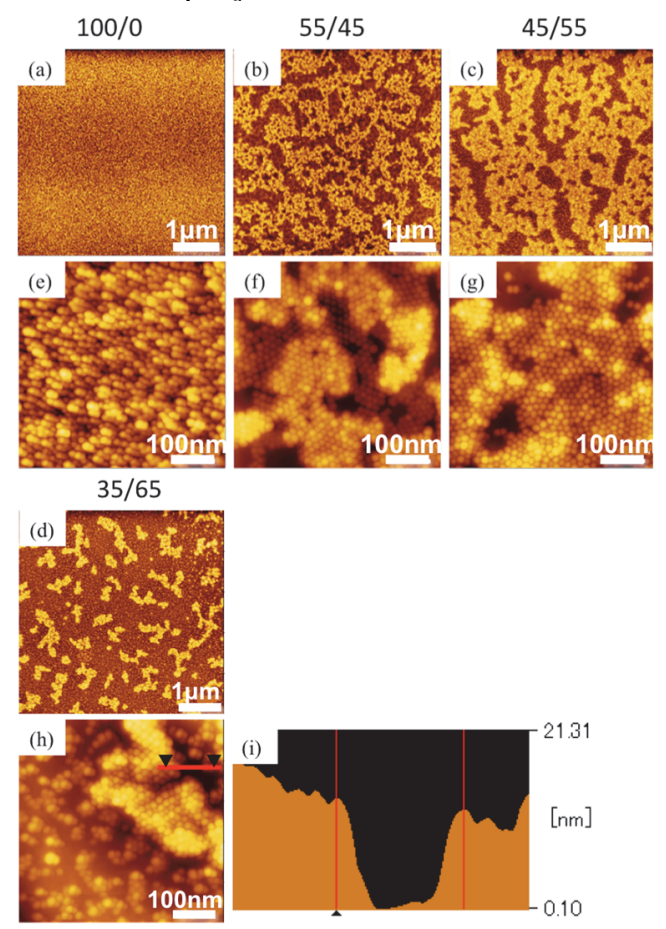

Figure 1. AFM height images of PS- $b$-PMAA/PAA (a) 100:0 (w/w), (b) 55:45 (w/w), (c) 45:55 (w/w), (d) $35: 65(\mathrm{w} / \mathrm{w})$ spin-coated thin films. (e-h) are the corresponding height images of (a-d). (i) is the cross sectional image of the red line shown in (h).

\subsection{Effect of composite polymer solution concentration \\ We also explored the effects of changing the}


concentrations ( $1 \mathrm{wt} \%, 2 \mathrm{wt} \%, 3 \mathrm{wt} \%$ in solution) of the composite polymer solution on the domain periodicity in PS- $b$-PMAA/PAA 45:55 (w/w) thin films. Certain defects and a terraced structure was observed in the AFM images (Figure 2a-c). We found that by increasing the composite polymer solution concentration from $1 \mathrm{wt} \%$ to $3 \mathrm{wt} \%$, the density of defects decreased and a packed structure could be observed with an increase in the film thickness. Thus, a $3 \mathrm{wt} \%$ solution was optimal for obtaining a well-ordered thin film with minimal defects and smaller thickness.

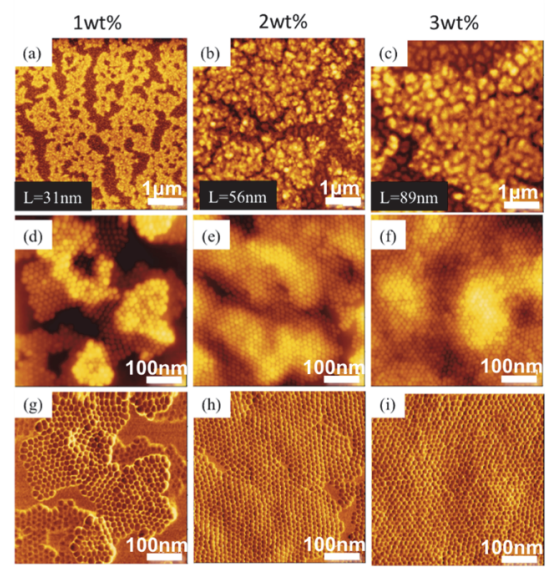

Figure 2. AFM height images of PS- $b$-PMAA/PAA 45:55 (w/w) thin films spin-coated from composite solution concentrations of (a) $1 \mathrm{wt} \%$, (b) $2 \mathrm{wt} \%$, (c) 3 $\mathrm{wt} \%$ in solution respectively. (d-f), (g-i) are the corresponding height and phase images of (a-c).

\subsection{Effect of heat treatment}

Thermogravimetric analysis (TGA) (Figure 3) was employed to monitor the degradation behavior of the block copolymers (red line) and composite polymers (PS-b-PMAA/PAA 35:65 $(\mathrm{w} / \mathrm{w})$ ) (black line) in a nitrogen atmosphere. Three detectable weight loss steps appeared during the analysis. Compared to the curve obtained from the analysis of PS- $b$-PMAA, a weight loss of ca. $8.5 \%$ was observed in the first temperature range of $100-254{ }^{\circ} \mathrm{C}$, suggesting that imidization occurred gradually. A weight loss of approximately $2 \%$ is expected for the dehydration of PAA in the imidization process. A $2 \%$ weight loss can also be found in the same temperature region of the TGA curve of pure PS- $b$-PMAA (weight ratio of 80:20). This is a result of the dehydration of PMAA as it forms a cross-linked network [5]. The remaining weight loss of ca. $6.5 \%$ observed in the TGA curve is likely due to the evaporation of residual solvent and the dehydration of the PMAA moiety. Weight loss was once again observed in the $350-500{ }^{\circ} \mathrm{C}$ range. This is mainly attributed to the degradation of the BCP template, and the spontaneous formation of porous structure in the polyimide matrix. The weight loss between $400{ }^{\circ} \mathrm{C}$ and 600 ${ }^{\circ} \mathrm{C}$ coincided with the shrinking of the polymer framework.

Based on the AFM analyses, a slow rate of heating is needed in order to minimize the rate of structural deformation of the films as the plasticity of the PS- $b$-PMAA increases (Glass Transition Temperature, $T_{\mathrm{g}, \mathrm{PS}}=102{ }^{\circ} \mathrm{C}$, Differential Scanning Calorimetry (DSC) data).

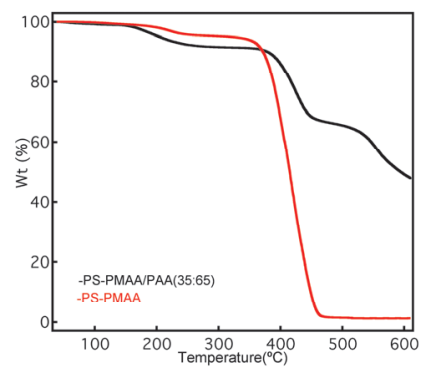

Figure 3. TGA curves for PS- $b$-PMAA and composite polymer of PS- $b$-PMAA/PAA 35:65(w/w).

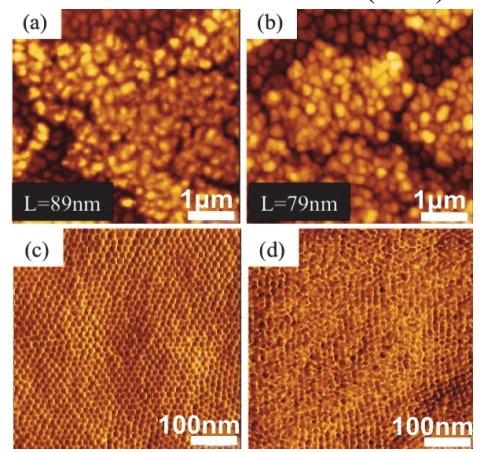

Figure 4. AFM height images of PS- $b$-PMAA/PAA $35: 65(\mathrm{w} / \mathrm{w})(3 \mathrm{wt} \%$ in solution) thin films as cast (a), and after heat treatment: (b) $80{ }^{\circ} \mathrm{C}$ for $7 \mathrm{~h}$. (c, d) are the corresponding phase images of $(a, b)$.

The AFM images for the PS- $b$-PMAA/PAA 35:65 (w/w) complex before and after heat treatment are shown in Figure 4. As observed in the AFM images, it is highly probable that the spherical structures had partially collapsed upon heating to $80{ }^{\circ} \mathrm{C}$. As the solution concentration increased, self-assembly of the nanostructure was able to proceed, however it is likely that residual solvent remained captured within the nanostructure, making complete solvent removal difficult. This residual solvent decreased the stability of the spherical structure during solvent evaporation and caused a partial collapse of the nanostructure.

This problem can be mitigated using crosslinkers to stabilize the ordered structure at moderate temperatures before the $\mathrm{BCP}$ diffuses out of the PAA matrix. Methanesulfonic acid was 


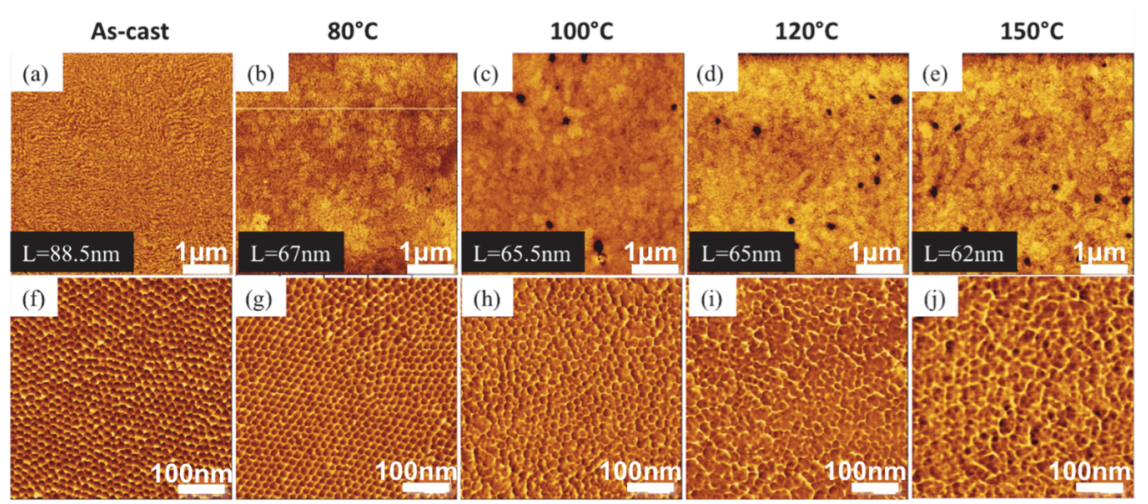

Figure 5. AFM height images of PS- $b$-PMAA/PAA/resol 35:55:10 (w/w) (3 wt $\%$ in solution) thin films at (a) as cast, and heat treatment: (b) $80{ }^{\circ} \mathrm{C}$ for $7 \mathrm{~h}$, (c) $100{ }^{\circ} \mathrm{C}$ for $12 \mathrm{~h}$, (d) $120^{\circ} \mathrm{C}$ for $30 \mathrm{~min}$, (e) $150{ }^{\circ} \mathrm{C}$ for $30 \mathrm{~min}$. (f-j) is the corresponding phase images of (a-e).

added to the solution [6] to catalyze the crosslinking reaction of the resol. A well-ordered spherical structure (PS- $b$-PMAA/PAA/resol 35:55:10 (w/w)) was successfully obtained in the thin film after curing at $80^{\circ} \mathrm{C}$. As the temperature increased, the film thickness decreased whereas the domain spacing remained constant. During imidization at $100{ }^{\circ} \mathrm{C}$, spherical structures were observed to have partially collapsed. However, upon the addition of the methanesulfonic acid, we were able to reduce the surface roughness of the thin films.

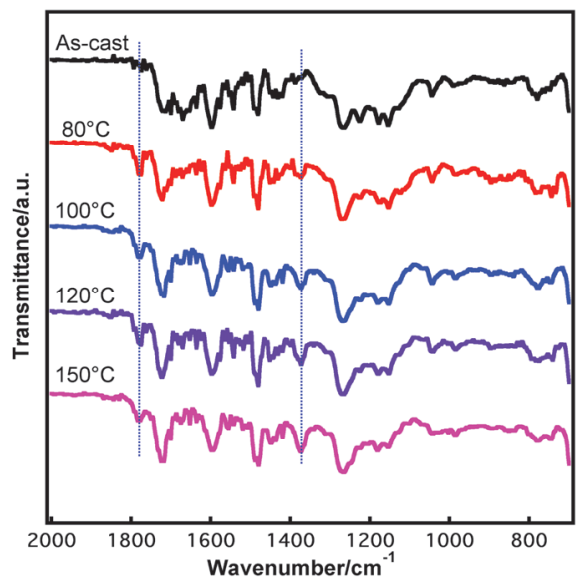

Figure 6. IR spectra of PS- $b$-PMAA/PAA/resol 35:55:10 (w/w) (3 $\mathrm{wt} \%)$ thin films after spin coating, $80{ }^{\circ} \mathrm{C}$ for $7 \mathrm{~h}, 100{ }^{\circ} \mathrm{C}$ for $12 \mathrm{~h}, 120^{\circ} \mathrm{C}$ for $30 \mathrm{~min}, 150$ ${ }^{\circ} \mathrm{C}$ for $30 \mathrm{~min}$, respectively.

IR spectra of the prepared polymers were obtained to confirm the formation of the polyimides after thermal treatment. The peaks at $1776 \mathrm{~cm}^{-1}(\mathrm{C}=\mathrm{O})$, and $1378 \mathrm{~cm}^{-1} \quad(\mathrm{C}-\mathrm{N}-\mathrm{C})$ corresponding to polyimide IR peaks appeared with increasing intensity as the temperature increased.

\section{Conclusion}

We successfully obtained well-ordered

spherical nanostructures in spin-casted films prepared by combining wholly aromatic condensation polymers and olefin type BCPs. No other structures were obtained despite increasing the ratio of PAA to $\mathrm{BCP}$. These results indicate that the micelle structures formed in solution were deposited onto the substrates to form the spherical nanostructures selectively. By increasing the concentrations of the solution used and adding methanesulfonic acid to catalyze the cross-linking reaction of the resol, it is possible to decrease the areal density of defects, such as terracing, and the surface roughness on the thin film. However, as the spherical structure had partially collapsed during imidization on the thin film as the temperature increased, further studies are required to obtain the optimal experimental conditions to obtain a stiff and well-ordered porous polyimide thin film.

\section{References}

1. H. Kosonen, S. Valkama, A. Nykänen, M. Toivanen, G. Brinke, J. Ruokolainen and O. Ikkala, Adv. Mater., 18 (2006) 201.

2. Y. Wan, Y. Shi and D. Zhao, Chem. Mater., 20 (2008) 932.

3. Y. Liu, K. Ohnishi, S. Sugimoto, K. Okuhara, R. Maeda, Y. Nabae, M. Kakimoto, X. Wang and T. Hayakawa, Polym. Chem., 5 (2014) 6452.

4. Y. Meng, D. Gu, F. Zhang, Y. Shi, L. Cheng, D. Feng, Z. Wu, Z. Chen, Y. Wan, A. Stein and D. Zhao, Chem. Mater., 18 (2006) 4447.

5. A. Asano, M. Eguchi, T. Kurotu, J. Therm, Anal. Cal., 56 (1999) 1059.

6. L. Sheng, T. Higashihara, R. Maeda, T. Hayakawa, M. Ueda, J. Polym. Sci., Part A: Polym, Chem., 51 (2013) 2216. 